\title{
Time-resolved flow field investigation in an industrial centrifugal compressor application involving TR-PIV synchronized with unsteady pressure measurements
}

\author{
J. Klinner ${ }^{1}$, M. Voges $^{1 *}$, M. Schroll ${ }^{1}$, A. Bassetti ${ }^{2}$, C. Willert ${ }^{1}$ \\ ${ }^{1}$ Inst. of Propulsion Technology, Engine Measurement Dept., German Aerospace Center (DLR), Köln, Germany \\ ${ }^{2}$ Inst. of Propulsion Technology, Acoustics Dept., German Aerospace Center (DLR), Berlin, Germany \\ *melanie.voges@dlr.de
}

Keywords: time-resolved PIV, correlation analysis, time-resolved pressure measurements, rotating instability

\begin{abstract}
We report on combined velocity and unsteady pressure measurements obtained on an radial compressor with vaneless diffuser and asymmetric volute. Time-resolved PIV recordings were acquired at $26 \mathrm{kHz}$ both upstream of the impeller as well as within the vaneless diffusor at several rotation speeds at clean conditions and prior to the onset of instabilities within the rotor. The velocity data was acquired with a high-repetition rate, double-pulse laser system consisting of two combined DPSS lasers and a high-speed CMOS camera that was synchronized with multi-point unsteady pressure measurements. Details on the facility, the utilized instrumentation and data processing are provided with particular focus on the spectral and coherence analysis. Power spectra obtained from time records of the inlet velocity and unsteady pressure reveal an increase of low-frequency fluctuations below the blade passing frequency and the occurrence of a mode-locked behaviour indicating the presence of rotating instabilities. High levels of correlation between velocity and unsteady pressure signals not only confirm the temporal coherence of the acquired data but also reveal a direct coupling between flow field and pressure signature that is more prominent upstream of the rotor rather than in the diffusor.
\end{abstract}

\section{Introduction}

The present investigation is aimed at capturing the signatures of instabilities in a centrifugal compressor that are reported to develop in the impeller (rotor) or vaneless diffuser or both (cf. (Sorokes et al., 2018)) when operated outside of nominal performance. The unsteady flow phenomena are believed to start from a localized flow separation (stall) that frequently will orbit within the stage at sub-harmonic rotational speeds (rotating stall or rotating instability, RI). If not damped sufficiently, the stall cells will eventually develop into more hazardous self-excited pressure oscillations within the entire compressor stage leading to surge phenomena that can induce high aero-elastic loads on the rotor itself leading to premature aging (fatigue) or ultimate failure of the stage. Predicting the onset of the instabilities is a prerequisite to maintain compressor operating within safe operating margins.

Although the application of conventional PIV techniques in rotating turbomachinery flows has reached state-of-the-art level (Wernet, 2000; Liu et al., 2006; Voges et al., 2013; Gancedo et al., 2016), very few studies are known in the current literature that investigate rotating stall using transient pressure measurements in combination with high speed or phase-locked PIV at high pressure ratios and operation conditions near the surge margin. Besides the challenging task to provide safer operation of the machine near surge in such experiments, advanced PIV methods in combination with unsteady pressure measurements can provide important insights into the formation of stall cells and the interactions between pressure and velocity fluctuations as well as the propagation of aeroacoustic modes in the machine (cf. (Pardowitz et al., 2015)).

The TR-PIV implementation presented herein follows the procedures described by (Willert, 2015), in which a narrow image domain is captured at high frame rates with large sample counts, typically at high 


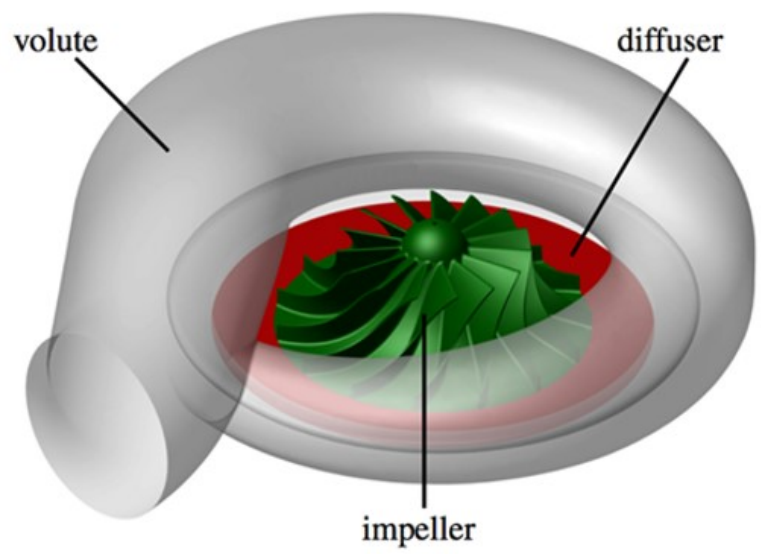

(a)

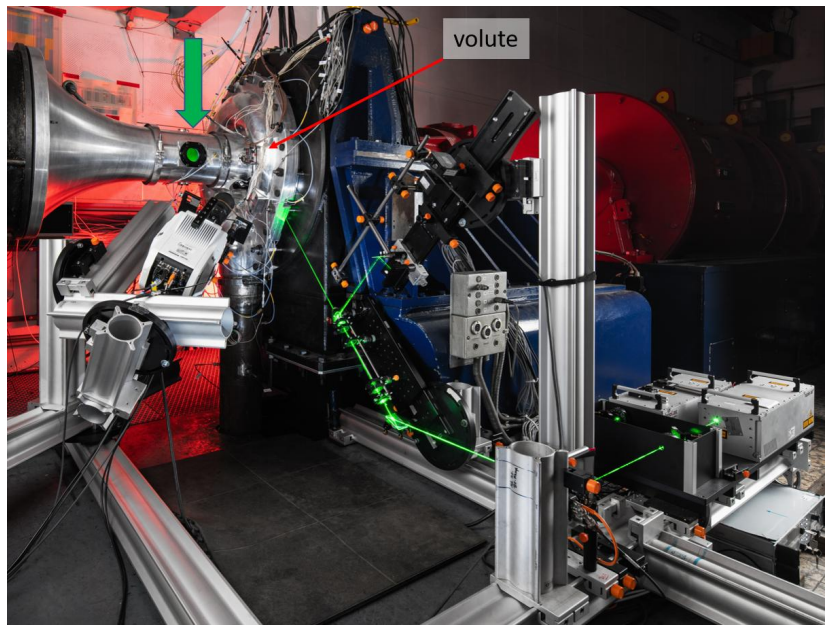

(b)

Figure 1: (a) Numerical model of the ROSSINI compressor stage with a vaneless diffuser and a volute (Rosemeier, 2017). (b) Photograph of the TR-PIV set up for investigation of the impeller exit flow, the arrow indicates the measurement location upstream of the impeller.

Table 1: ROSSINI compressor stage dimensions

\begin{tabular}{lrl} 
Number of Blades & 15 & \\
Shroud (inlet) radius $r_{s}$ & 79.37 & $\mathrm{~mm}$ \\
Impeller LE tip radius $r_{1}$ & 78.97 & $\mathrm{~mm}$ \\
Impeller TE tip radius $r_{2}$ & 127.84 & $\mathrm{~mm}$ \\
Diffuser exit radius $r_{3}$ & 214.13 & $\mathrm{~mm}$ \\
Diffuser passage height $h$ & 14.37 & $\mathrm{~mm}$ \\
Design speed & 23,900 & $\mathrm{rpm}$ \\
Pressure ratio, $\Pi$ & 1.9 & - \\
Mass flow, $\dot{m}$ & 2.8 & $\mathrm{~kg} / \mathrm{s}$ \\
\hline
\end{tabular}

image magnification, to provide time-records of a single column of velocity vectors evolving in time. The present work demonstrates the applicability of a recently developed high-repetition rate double-pulse laser system (Klinner et al., 2021) in a rotating machine.

The TR-PIV measurements in the inlet section upstream of the rotor were aimed at characterizing the properties of the inflow at the domain inlet for accompanying LES and URANS computations (not covered in the present contribution). A second TR-PIV measurement domain was located in the diffuser channel for investigation of the flow exiting the rotor. Data analysis was focused on time history of the signals, spectra and combined correlation analysis of the transient pressure and velocity information analogous to previously conducted measurements on axial compressor rigs (see (Pardowitz et al., 2014, 2015)).

\section{Description of test rig and centrifugal compressor}

The single-stage centrifugal compressor geometry provided by Liebherr Aerospace Toulouse SAS (LTS) consists of an impeller with 15 unshrouded blades in backswept design, a vaneless diffuser and an asymmetric volute, as shown in Fig. 1(a) In its original application the compressor stage is operated inside of the cabin air conditioning system of a civil aircraft. For the present investigation the original geometry was adapted at DLR to facilitate the integration of extensive instrumentation. At its design point the realized stage operates a rotational speed of about $23,900 \mathrm{rpm}$ and a total stage pressure ratio of $\Pi \approx 1.9$. The inlet and outlet sections consist of straight pipes. The characteristics of the compressor stage are given in Table 1 .

The performance map, shown in Fig. 2(a) provides six measured speed lines from $40 \%$ to $120 \%$ of the design rotational speed in comparison with selected CFD results of the numerical twin of the compressor 
stage as described in (Voges et al., 2021). The agreement between numerical and experimental results is very good, especially regarding the unsteady RANS calculations (black squared symbols in Fig. 2 .

Prior to the experimental study presented herein, a detailed investigation of the stability limit and surge behavior of the rig was performed. Online FFTs of the unsteady pressure probe signals were used to identify the beginning of unstable flow conditions and possible entry into rotating stall when throttling the flow, which is visible as a low-frequency 'hump' and 'ripples' in the spectra below the blade passing frequency (BPF) and above the rotor frequency $f_{\text {rotor }}$ (see Fig. 2(b) $)$. In comparison to clean conditions the 'hump' indicates an increase of broadband fluctuations below the BPF, whereby the 'ripples' indicate a further increase of fluctuations and some mode-locked behaviour and are seen as a precursor of a rotating instability (RI) (Voges et al., 2021; Pardowitz et al., 2015).

The operating conditions for combined TR-PIV and unsteady pressure array measurements at four different rotational speeds were chosen to correspond to either conditions where instabilities occur (i.e. 'hump' or 'RI'), or clean reference conditions as indicated by the white circles in the performance map (Fig. 2(a)).

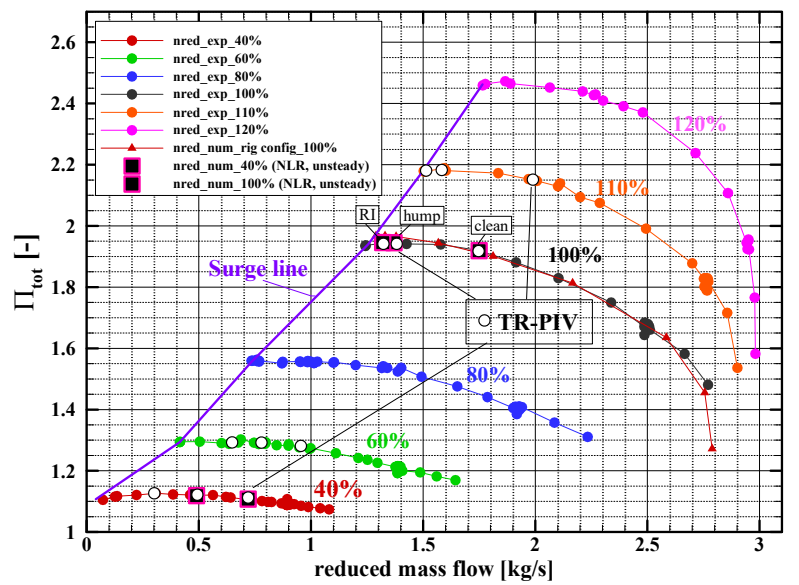

(a)

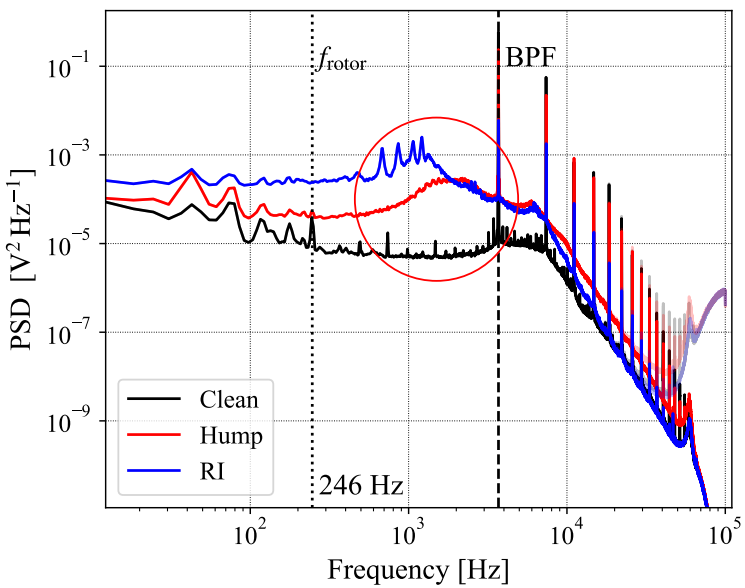

(b)

Figure 2: (a) Performance map of the ROSSINI rig. Solid circles represent data from experiment, solid diamonds from RANS and solid squares from URANS simulation. White circles indicate conditions for TR-PIV measurements. (b) Typical spectra for clean and unstable conditions at $60 \%$ speed calculated from a unsteady pressure signal of the rig used for setting up 'clean', 'hump' and 'RI' conditions.

\section{Unsteady pressure measurements and transient recording of signals}

To enable spatio-temporal analyses of the unsteady pressure fluctuations in the centrifugal compressor rig (Pardowitz et al., 2014, 2015), time-resolved pressure array measurements were recorded using 19 miniature unsteady pressure transducers (Kulite, XCE-062) that were flush-mounted in the impeller casing. These sensors were arranged in an asymmetric distribution at irregular circumferential positions, that is, their positioning does not follow the same periodicity when considering characteristic modal structures of unsteady phenomena related to stall and its precursors. The sensors are grouped to form arrays at 3 different meridional positions (see Fig. 3 and Table 2).

Depending on pressure amplitude the pressure transducers have a total accuracy to capture unsteady fluctuations least of 0.07 mbar increasing to $0,12 \mathrm{mbar}$ at 3.5 bar (full scale).Using a Dewetron 808 data acquisition system with a DEWE-ORION-0824 multichannel 24 bit AD-converter the unsteady pressure signals were acquired at a sample rate of $200 \mathrm{kHz}$, with a $-3 \mathrm{db}$ bandwidth of $150 \mathrm{kHz}$ for the relevant measurement range. The data acquisition system also recorded the rig's spindle speed, PIV image acquisition triggers, several microphone signals and gap sensors. To synchronize record start times across multiple measurement sequences, a satellite time reference (IRIG-B) was also acquired with each transient recording. 


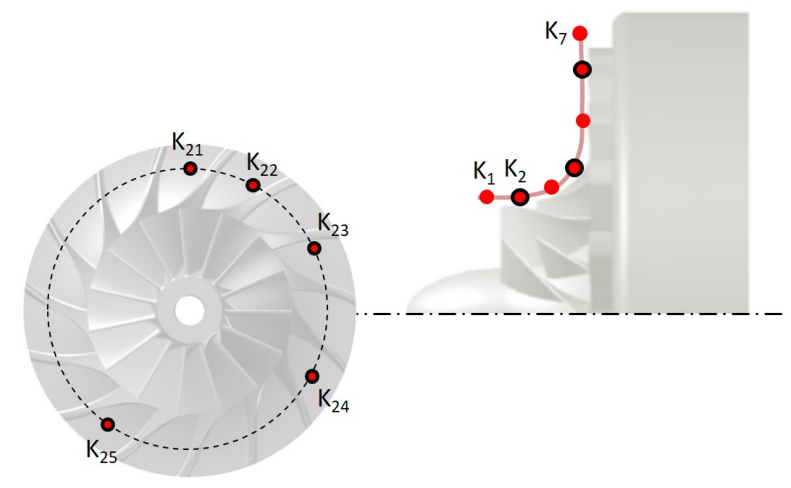

Figure 3: Positioning of the unsteady pressure probes (Kulites) on the impeller casing.

Table 2: Kulite array positions on the impeller casing

\begin{tabular}{llll}
\hline Kulite sensor & $\begin{array}{l}\text { Position } \\
x / s_{m}[\%]\end{array}$ & $\begin{array}{l}\text { start angle } \\
{\left[^{\circ}\right]}\end{array}$ & $\begin{array}{l}\text { angle increments } \\
{\left[{ }^{\circ}\right]}\end{array}$ \\
\hline Impeller inlet $\mathrm{K}_{1}$ & -10 & 190 & \\
Array $1 \mathrm{~K}_{21}-\mathrm{K}_{25}$ & 5 & 190 & $27 ; 36 ; 43 ; 115$ \\
Impeller $\mathrm{K}_{3}$ & 25 & 195 & \\
Array $2 \mathrm{~K}_{41}-\mathrm{K}_{45}$ & 50 & 190 & $27 ; 36 ; 43 ; 115$ \\
Impeller $\mathrm{K}_{5}$ & 75 & 200 & \\
Array $3 \mathrm{~K}_{61}-\mathrm{K}_{65}$ & 95 & 205 & $27 ; 36 ; 43 ; 115$ \\
Impeller $\mathrm{K}_{7}$ & 110 & 170 & \\
\hline
\end{tabular}

\section{TR-PIV instrumentation and image evaluation}

With the aim of measuring both the upstream and downstream propagation of RI-induced disturbances, planar two-component high-speed PIV (2D 2C TR-PIV) was performed at two locations (Fig. 4). One measurement area (location \#1) is located in center of the intake tube $3.0 r_{1}$ upstream of the impeller leading edge (LE), while the location \#2 is positioned in the diffuser immediately downstream of the impeller exit at $1.1 r_{2}$.

Velocity magnitudes up to $70 \mathrm{~m} / \mathrm{s}$ at the impeller inlet and up to $350 \mathrm{~m} / \mathrm{s}$ at the outlet required the use of a double-pulse high speed laser system, which enables pulse separations in the required regime between $1-20 \mu \mathrm{s}$ at repetition rates of double pulses of $f_{s}=26 \mathrm{kHz}$. The laser system consists of a pair of diodepumped solid state lasers (Innolas Photonics, Nanio Air 532-10-V-SP), each providing an average power of up to $10 \mathrm{~W}$. Further details on the above TR-PIV instrumentation can be found in Klinner et al. (2021). An in-house beam-combining optics superimposes the beams from the resonators into a common beam which is then collimated into a narrow, $2.5 \mathrm{~mm}$ wide light-sheet with a thickness between $250 \mu \mathrm{m}$ (location \#1) and $450 \mu \mathrm{m}$ (location \#2). The thickening of the light-sheet at the latter location was necessary to minimize the loss-of-pairs in the turbulent impeller exit flow. Dense smoke oil-based seeding was provided by a smoke generator (Vicount) into the intake section of the test rig through a centrifugal pump and settling chamber to facilitate aerosol sizes $<1 \mu \mathrm{m}$. The light scattered by the tracers was imaged with a CMOS high speed camera (Vision Research, Phantom v1840) camera using frame-straddling at a frame rate of $54 \mathrm{kHz}$. To improve statistical convergence, two runs were recorded per rig test point each containing 8 bursts at $0.5 \mathrm{~Hz}$ repetition, which each burst is containing 10,642 double images recorded at 26,000 image pairs per second $(\sim 0.4 s)$.

The camera was equipped with a macro lens (Nikon, Nikkor Micro f200/4) with a magnification set near unity which enabled imaging ratios of $11.6 \mu \mathrm{m} / \mathrm{pixel}$ at location \#1 (inlet) and of $13.3 \mu \mathrm{m} / \mathrm{pixel}$ at location \#2 (impeller exit, inside the diffuser). While location \#1 had optical access through a glass window in the inlet tube with an overall working distance of $220 \mathrm{~mm}$, location \#2 required a complex imaging setup via a $125^{\circ}$ mirror and a window, thus requiring a larger working distance of $250 \mathrm{~mm}$. The longer stand-off distance resulted in a slightly lower spatial resolution (see Fig. 1(b). At frame rates of $54 \mathrm{kHz}$ the highspeed camera provided reduced image sizes of $1792 \times 160$ pixel, which corresponded to an image area of 


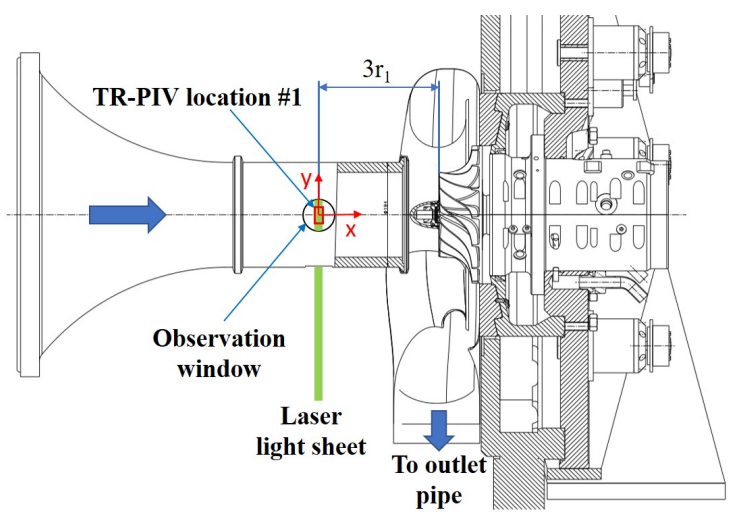

(a) Inlet configuration

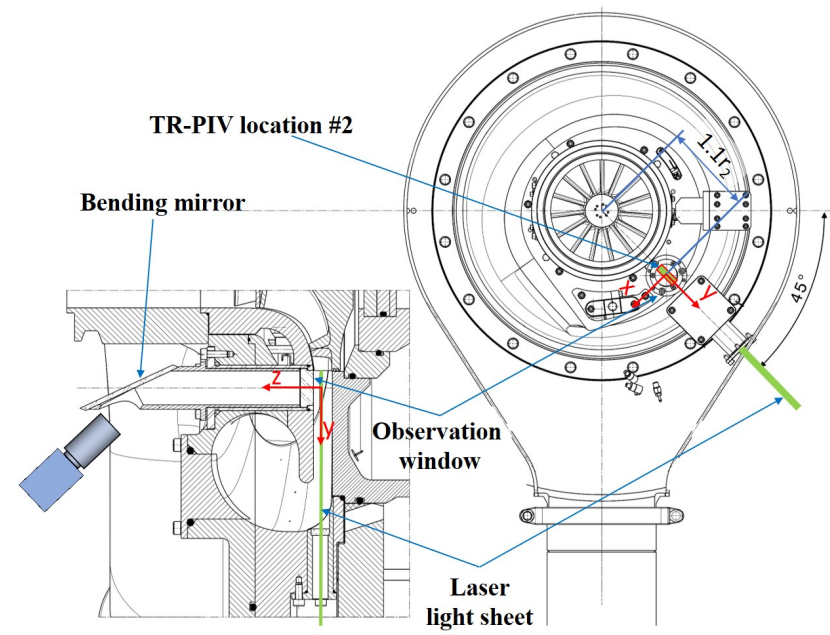

(b) Impeller exit configuration

Figure 4: Measurement stations for TR-PIV.

$20.78 \times 1.85 \mathrm{~mm}^{2}$ at location \#1 and of $23.9 \times 2.1 \mathrm{~mm}^{2}$ at location \#2.

Inlet flow particle images recorded at location \#1 (cf. Fig. 4(a) exhibit a very high particle image density and allow for high spatial sampling of PIV analysis. Thus, using state-of-the-art PIV processing techniques, a final interrogation window sizes of $16 \times 16$ pixel $\left(190 \times 190 \mu \mathrm{m}^{2}\right)$ with validation rates of $100 \%$ were feasible for all operation conditions. The corresponding validation scheme involves a normalized median filter with a threshold of 3.0. At the downstream location \#2, the increased turbulence levels required the use of a larger sampling window size of $32 \times 32$ pixels $\left(430 \times 430 \mu \mathrm{m}^{2}\right)$ for the $40 \%$ RI condition. With increasing rotational speeds the window size was further increased to $64 \times 32$ pixels $\left(860 \times 430 \mu \mathrm{m}^{2}\right)$, which is due to the increased tangential velocity along with higher fluctuations. Using a normalized median filter with a threshold of 3.0, validation rates of at least $95 \%$ and typically of $98-99 \%$ could be achieved for measurements at the impeller exit.

To enhance particle image contrast and thereby improve the cross-correlation analysis, a mean intensity image, calculated from each burst's image sequence, was subtracted. All particle images were evaluated using commercial software (PIVview 3.9) and an in-house Python-based PIV package.

\section{Results}

\subsection{Time-resovled velocity records}

Fig. 5 shows time traces of the axial $(u)$ and transverse $(v)$ velocity components on the inlet center-axis. The shown single burst of $0.4 \mathrm{~s}$ length were recorded at $100 \%$ rotational speed at both clean and disturbed (throttled) conditions. As the flow is throttled to 'hump' conditions, the mean axial inlet velocity $\bar{u}$ decreases and is accompanied with an increasing occurrence of low-frequency modulations of the axial velocity $u$. When 'RI' conditions are reached, the axial velocity exhibits a nearly sinusoidal modulation near $40 \mathrm{~Hz}$ with amplitudes of about $\pm 10 \%$ of the mean, which believed to be directly associated with presence of a single rotating stall cell inside of the stage. It should be noted, that the transverse velocity component $(v)$ exhibits no such signature.

At measurement location \#2 (Fig. 4(b)), the laser light sheet was periodically scattered by the impeller blades leading to over-exposed image background intensities with low particle image visibility (poor signalto-noise ratio) and drop-out in the velocity records. At $40 \%$ speed this affected about $10 \%$ of the PIV samples, increasing to $26 \%$ at $110 \%$ speed.

Fig. 6 presents exemplary velocity time records of the impeller exit flow at $60 \%$ speed. Each column in the plot represents the circumferential velocity measured in the central column of successive PIV samples. As indicated in Fig. 4(b), the $y$-axis is aligned with the radial direction originating at the machine axis. PIV data affected by strong laser flare due to the light scattered from the impeller blading could be reliably 

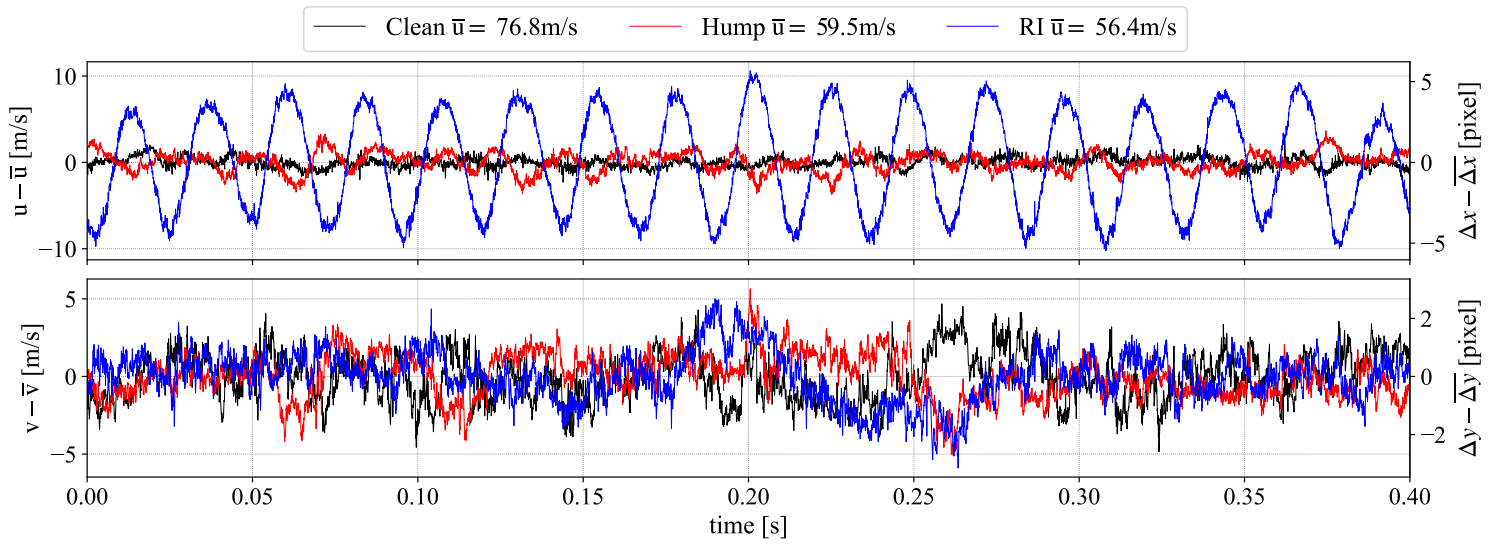

Figure 5: Time traces of axial (top) and transverse (bottom) velocity at location \#1 and $y=0$ at $100 \%$ speed showing increasing low-frequency modulations of the axial velocity at 'hump' and 'RI' conditions. With the present scaling a displacement of 0.1 pixel corresponds to approx. $\pm 0.2 \mathrm{~m} / \mathrm{s}$.

identified by imposing a threshold in the circumferential (i.e. tangential) velocity $u_{i}<0.3 \bar{u}$. Furthermore, frames strongly affected by laser reflections were rejected when the total validation rate per frame dropped below a threshold of $70 \%$. This results in blanked (white) columns in Fig. 6 Due to aliasing between the BPF and image sample rate, this blanking is not equidistant such that certain blade passages remain unaffected.

When comparing circumferential velocity variations of the exit flow over time, Fig. 6(top) shows more or less a homogeneous spatial distribution for 'clean' conditions and seems uncorrelated with the phase angle of blade passing. In contrast, 'hump' (middle) and 'RI' (bottom) conditions exhibit a clear deficit of tangential velocity after blade tip arrival (probably the blade's wake flow) which is followed by a region of increased circumferential velocity shortly before the following blade arrives (indicated by the following white bar). For 'hump' and 'RI' conditions, these regions of increased circumferential velocity appear at the impeller exit $(y=-10 \mathrm{~mm})$ and propagate radially outward with time, as indicated in the dashed line in Fig. 6 (middle).

\subsection{Spectral analysis of the inlet flow}

The power spectral density (PSD) of the inlet velocity data was obtained using Welsh's method (Welch, 1967) by summing of Hann-weighted FFTs accumulated in equi-spaced slots of interval $4096 / f_{s}(157 \mathrm{~ms})$ with $2730 / f_{s}$ (105 ms) spacing (66\% overlap), corresponding to a spectral binwidth of $6.35 \mathrm{~Hz}$. Lowpass filtering with a cut-off of $0.4 f_{s}$ was applied to attenuate random noise. The spectra were additionally averaged over the eight bursts to improve statistical convergence. For the inlet flow, the interrogation window size was varied between 16, 32 and 64 pixel which was found to have very little effect on the shape of the spectrum. Also, shape variations of the spectra in the transverse $y$ direction over the $21 \mathrm{~mm}$ image height (see Fig. 4(a) are marginal, thus allow averaging along the extent of the field of view to further decrease random noise.

Fig. 7 (left) shows the resulting velocity spectra recorded at $100 \%$ rotor speed for the three throttle positions. The $-5 / 3$ slope is added to indicate Kolmogorov's scaling law which seems to partially apply to the transverse component. With increased throttling of the stage at constant rotor speed there is strong increase in harmonic components below the rotor frequency for the axial component $(u)$ which results in a maximum near $44 \mathrm{~Hz}$ for 'RI' conditions that already was clearly visible in the corresponding time trace in Fig. 5. This is accompanied by additional spikes present between BPF and $f_{r}$ for the spectra of the transverse velocity. Integration of the spectra confirms an increase of turbulence intensity $u^{\prime} / \bar{u}$ from $0.8 \%$ at 'clean' conditions to $2 \%$ at 'hump' conditions up to $10 \%$ at 'RI'. The latter increase of the standard deviation $u$ ' stems from the strong low-frequency modulation, also visible in the time trace show in Fig. 5 (top). A comparison to PSDs of the unsteady pressure signals obtained at the rotor inlet $\left(\mathrm{K}_{1}\right)$ in Fig. 7 (right) reveals similar spikes near $40 \mathrm{~Hz}$ as in the $u$-PSD which were also used to verify the temporal coherence between the pressure and velocity signals and thereby confirm synchronized data acquisition. This is described in the following section. 


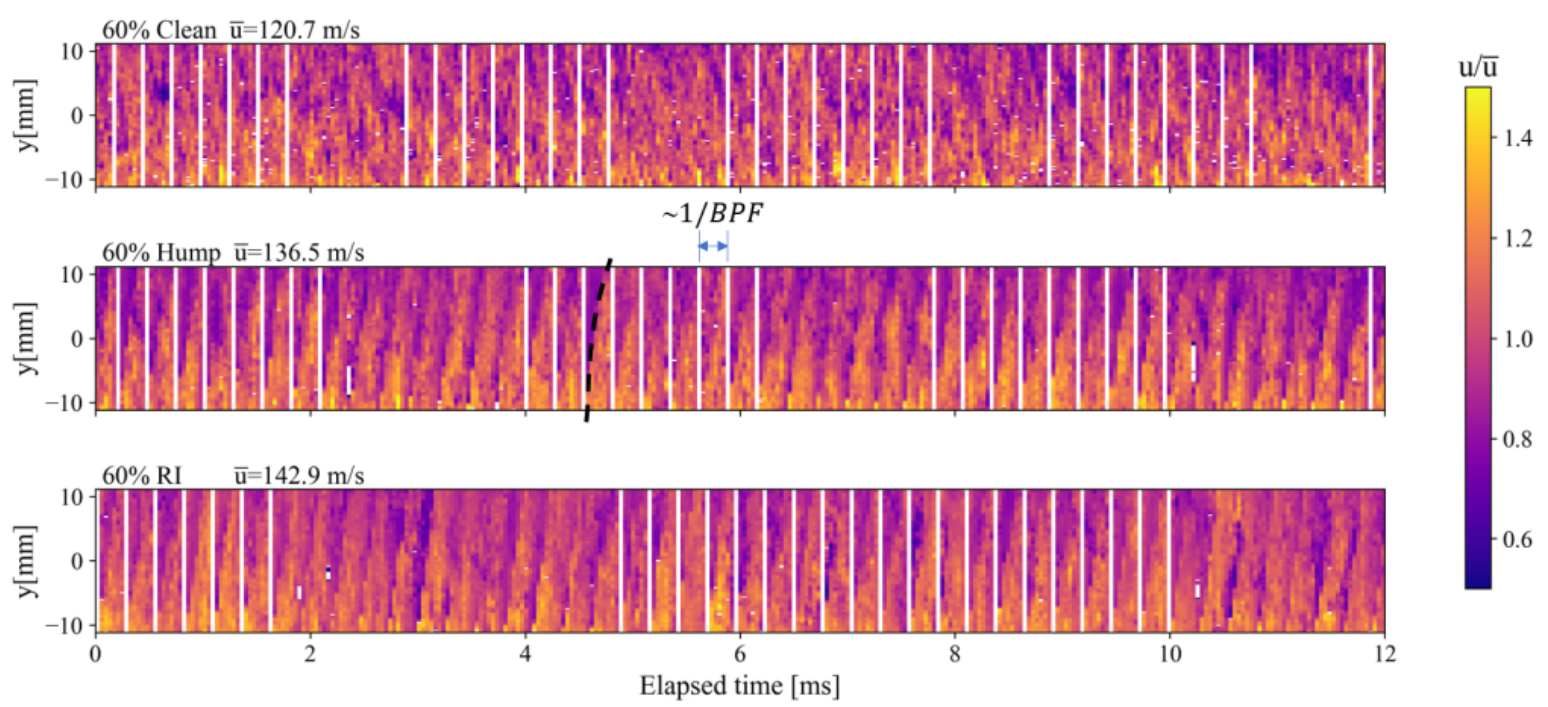

Figure 6: PIV timetraces of circumferential velocity $u$ of the impeller exit flow and along the radial direction $y$ recorded with $26 \mathrm{kHz}$ at location \#2 near the casing wall at 'clean' (top), 'hump' (middle) and 'RI' (bottom) conditions. White bars represent measurements affected by laser reflections on the impeller blades.

\subsection{Velocity-pressure correlations and verification of phase coherence}

For the present time-resolved database, velocity-pressure correlations and evaluations of co-spectra are feasible on the basis the measurements were acquired synchronously. To verify the consistency, the start-triggers of each of the PIV burst was recorded by the data acquisition system described in Sec. 3. These signals are automatically detected in the transient recordings by first binarizing the signal at a threshold of half of the TTL voltage level. In a second step the position was detected were the gradient of the binarized signal is half of the TTL level. This allowed the absolute positioning each PIV sequence with a jitter in the range of $[-5,+15 \mu \mathrm{s}]$ with respect to the time-base associated with the pressure signals.

While PIV validation rates of $100 \%$ were achieved for the inlet measurements, the outlet measurement data is incoherent due the laser flare caused by the passing blades and first needs to be interpolated to obtain equidistant samples and to enable correlations. For this purpose, a third order cubic spline interpolation was applied using the velocity data from previous and subsequent PIV samples.

The signal coherence between pressure and PIV data could be clearly verified at the $100 \%$ speed 'RI' test point. Here the presence of the previously mentioned modulation near $44 \mathrm{~Hz}$ is present in both the pressure and the velocity signal (see Fig. 7 bottom). Fig. 8 shows the resulting associated time traces for pressure and velocity data for inlet measurements (left) and outlet measurements (right) for a single burst. The unsteady pressure data are equivalent to those shown in the spectra in Fig. 7 and were downsampled from $200 \mathrm{kHz}$ to $26 \mathrm{kHz}$ through linear interpolation to obtain a common time base. Velocities represent the value averaged over the $y$-axis excluding outliers, where outliers due to blade reflections were interpolated after spatial averaging. The $40 \mathrm{~Hz}$ modulation is clearly visible in the inlet measurements but is attenuated in the outlet measurements. For better visualization, Fig. 8 also includes a low-pass filtered version of velocity signal (red line) at the outlet with cut-off at the rotor speed (1/15th of BPF). By comparing these combined plots of pressure and velocity for all of the eight burst per run, the temporal coherence between the pressure and velocity could be verified.

Cross-correlation between the spatially averaged velocities and the unsteady pressures at the rotor inlet and outlet are shown in Fig. 9 for 100\% speed at 'RI' conditions. Each correlation is averaged over eight bursts and reveals clearly a dominant sinusoidal modulation near $44 \mathrm{~Hz}$ as visible in unsteady pressure and velocity spectra. Interestingly, the correlation coefficient is much weaker for the exit velocity which probably means that, under given operation conditions near surge, wall pressure fluctuations have a greater influence on the velocity field upstream of the rotor than on the velocity in the diffuser. 

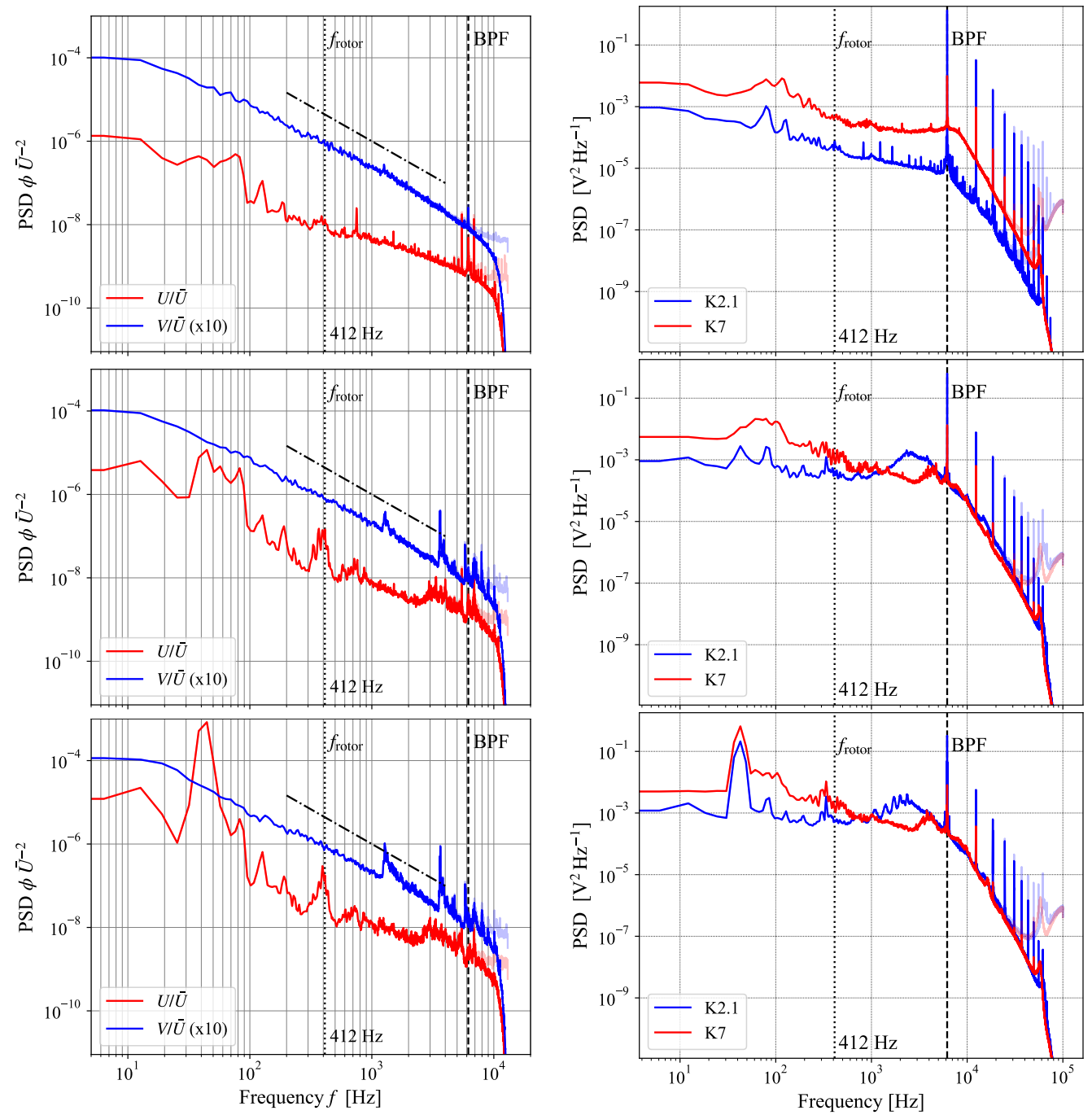

Figure 7: PSDs of velocities (left) measured at location \#1 $(y=0)$ and PSDs of unsteady pressure (right) on impeller casing entry at $x / s_{m}=5 \%\left(\mathrm{~K}_{21}\right)$ and immediately downstream of the rotor at $x / s_{m}=110 \%\left(\mathrm{~K}_{7}\right)$ at $100 \%$ speed and at 'clean' (top), 'hump' (middle) and 'RI' (bottom) conditions. Faded colors represent the raw un-filtered data.

\section{Conclusions}

The present contribution describes the implementation of TR-PIV synchronized with unsteady pressure measurements in an industrial single-stage centrifugal compressor. The rig was operated at four speed lines near design point and near surge in order to provide experimental data with regard to the precursors of instabilities or even stall. In total approximately 3 Terabytes of particle image data were acquired at two measurement locations, one upstream of the impeller leading edge and the other immediately downstream of impeller exit within the vaneless diffuser. The analysis of power spectra for inlet velocities and unsteady pressure data located at $5 \%$ and $110 \%$ of meridional span revealed the increase of low-frequency fluctuations below the BPF as well as below the rotor frequency and the occurrence of a mode-locked behaviour ('ripples') indicating the presence of rotating instabilities near surge. Exemplarily correlations between measured velocity and unsteady pressures revealed consistency of the data in terms of a large correlation signal at the dominant low-frequency peak at $100 \%$ speed in both pressure and velocity spectra. Future work could make use of measured phase differences of such correlations to compute propagation velocities and direction of perturbations in the velocity field related to unsteady surface pressure. The available data is suitable for Fourier decomposition of the signals obtained by unsteady pressure array which can provide time-resolved 
information on the occurrence of rotating instabilities in the impeller casing and which, ideally, can be used for further conditional analysis of the flow field data provided by TR-PIV.
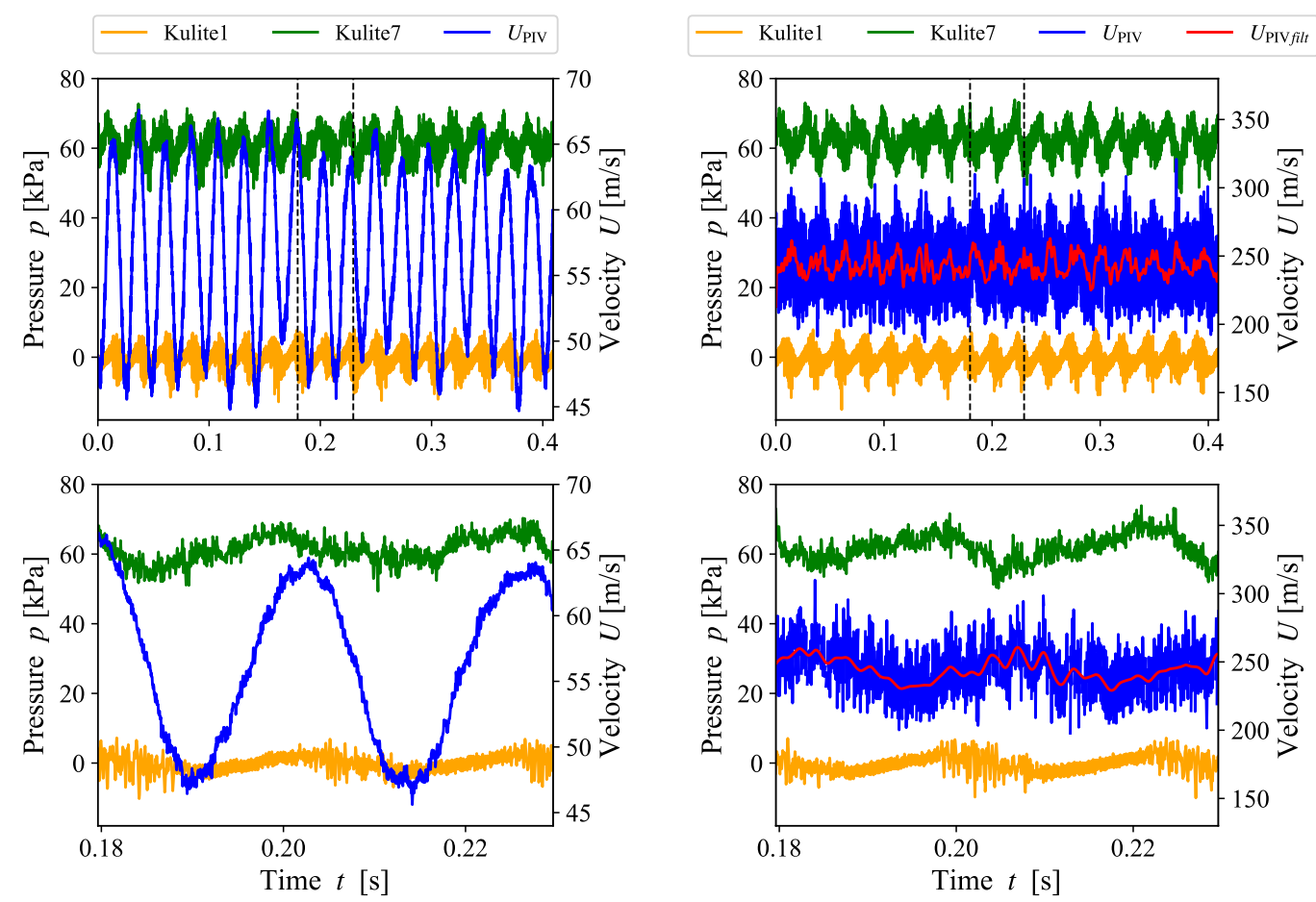

(a)

(b)

Figure 8: Time traces at 100\% speed 'RI' of unsteady pressures of two Kulites at inlet and impeller exit and axial velocity measured at inlet location \#1 (a) and radial velocity measured at impeller outlet location \#2 (b). Top: entire burst; Bottom: Enlarged section between the dashed lines.

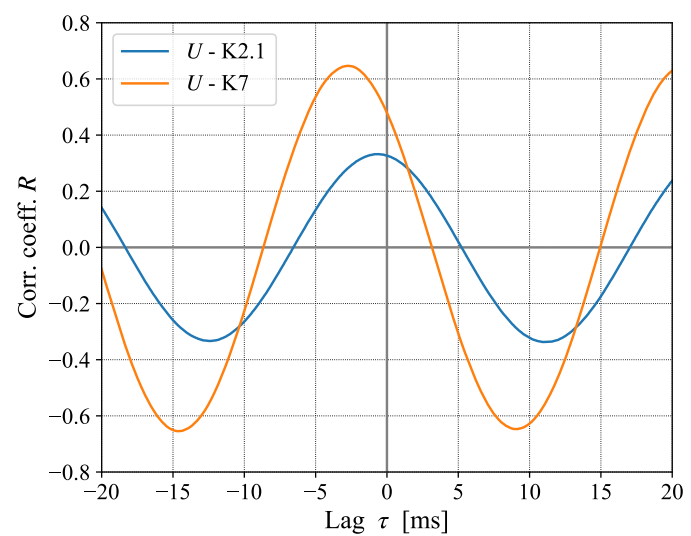

(a)

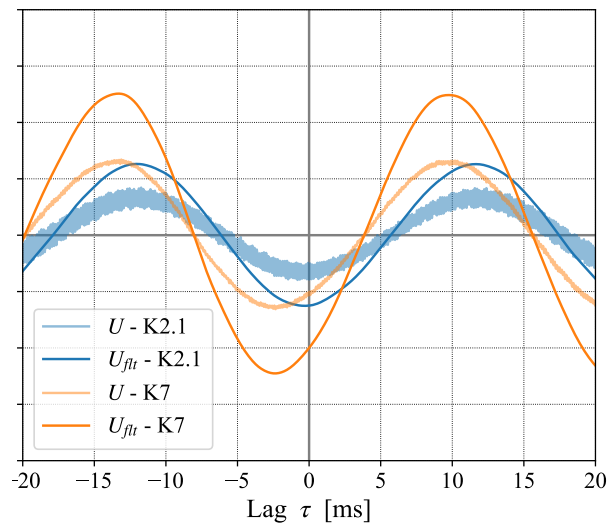

(b)

Figure 9: Cross-correlations of velocities of the inlet flow at location \#1 (a) and the impeller exit flow at location \#2 (b) at $100 \%$ speed 'RI' correlated with unsteady pressure signals on impeller casing entry at $x / s_{m}=5 \%\left(\mathrm{~K}_{21}\right)$ and immediately downstream of the rotor at $x / s_{m}=110 \%\left(\mathrm{~K}_{7}\right)$. 


\section{Acknowledgements}

The material presented herein was funded through the Clean Sky 2 Joint Undertaking Project ROSSINI (Radial cOmpreSsor Surge INception Investigation) under the European Union's Horizon 2020 research and innovation program. (grant agreement No 717081). The authors gratefully acknowledge the contributions of all project team members, namely Mr. Schindel and the workshop team from DLR, for their effort and valuable contribution during rig design and instrumentation phase.

\section{References}

Gancedo M, Gutmark E, and Guillou E (2016) PIV measurements of the flow at the inlet of a turbocharger centrifugal compressor with recirculation casing treatment near the inducer. Experiments in Fluids 57:16

Klinner J, Hergt A, Grund S, and Willert CE (2021) High-Speed PIV of shock boundary layer interactions in the transonic buffet flow of a compressor cascade. Experiments in Fluids 62:58

Liu B, Yu X, Liu H, Jiang H, Yuan H, and Xu Y (2006) Application of SPIV in turbomachinery. Experiments in Fluids 40:621-642

Pardowitz B, Peter J, Tapken U, Thamsen PU, and Enghardt L (2015) Visualization of Secondary Flow Structures Caused by Rotating Instability: Synchronized Stereo High-Speed PIV and Unsteady Pressure Measurements. in 45th AIAA Fluid Dynamics Conference. American Institute of Aeronautics and Astronautics, Dallas, TX

Pardowitz B, Tapken U, Sorge R, Thamsen PU, and Enghardt L (2014) Rotating Instability in an Annular Cascade: Detailed Analysis of the Instationary Flow Phenomena. Journal of Turbomachinery 136:061017

Rosemeier J (2017) Numerische Analyse eines Radialverdichters mit Spiralgehäuse und unbeschaufeltem Diffusor. Master's thesis. Ruhr Universität Bochum

Sorokes JM, Marshall DF, and Kuzdzal MJ (2018) A review of aerodynamically induced forces acting on centrifugal compressors and resulting vibration characteristics of rotors. in 47th Turbomachinery and 34th Pump symposia. Houston, Texas

Voges M, Klinner J, Willert C, Bassetti A, Reutter O, and van Rooij M (2021) The Challenge of Time-Resolved Flow Investigation of a One-Stage Centrifugal Compressor with a Non-Symmetric Volute. in 14th European Turbomachinery Conference. Gdansk, Poland

Voges M, Willert C, Mönig R, and Schiffer HP (2013) The Effect of a Bend-Slot Casing Treatment on the Blade Tip Flow Field of a Transonic Compressor Rotor. in Proceedings of the ASME Turbo Expo, San Antonio, Texas, USA

Welch P (1967) The use of fast fourier transform for the estimation of power spectra: A method based on time averaging over short, modified periodograms. IEEE Transactions on Audio and Electroacoustics 15:70-73

Wernet MP (2000) A flow field investigation in the diffuser of a high-speed centrifugal compressor using digital particle imaging velocimetry. Measurement Science and Technology 11:1007-1022

Willert CE (2015) High-speed particle image velocimetry for the efficient measurement of turbulence statistics. Experiments in Fluids 56:17 